\title{
Une vie de ver
}

Nathalie Pujol, Jonathan J. Ewbank

> Malgré sa simplicité anatomique, le nématode Caenorhabditis elegans (C. elegans) est un organisme multicellulaire complexe. Au travers des études qui ont contribué à une meilleure compréhension de certains aspects de la physiologie du ver, nous présentons dans cette revue les éléments concernant les bases cellulaires et moléculaires des interactions entre $C$. elegans et son environnement, notamment les capacités sensorielles, l'horloge biologique interne qui gouverne la vitesse de développement du ver, et certains facteurs contrôlant sa longévité. Nous exposons aussi brièvement les résultats très récents qui démontrent l'existence d'une immunité innée chez ce nématode. Finalement, nous soulignons certaines méthodologies récentes qui ouvrent la voie à des études fonctionnelles systématiques chez C. elegans. <

Dans cet article qui ne se veut pas exhaustif, nous présentons certains aspects de la vie et de la physiologie du nématode Caenorhabditis elegans (C. elegans). Ainsi, nous ne traiterons pas des mécanismes d'apoptose, ou de la reproduction. La monographie « C. elegans ||$»$, très complète sur l'ensemble des divers aspects de la vie du nématode, est un bon point de départ [1]. Elle est maintenant en libre accès sur Internet (http://www.ncbi.nlm.nih.gov/books/), de même qu'un atlas interactif décrivant l'anatomie du ver (http://www.wormatlas.org/).

\section{À la recherche de nourriture}

Comme toutes les espèces, pour vivre et se reproduire, le ver doit manger. Les vers vivent dans le sol où ils puisent les bactéries dont ils se nourrissent, grâce à un

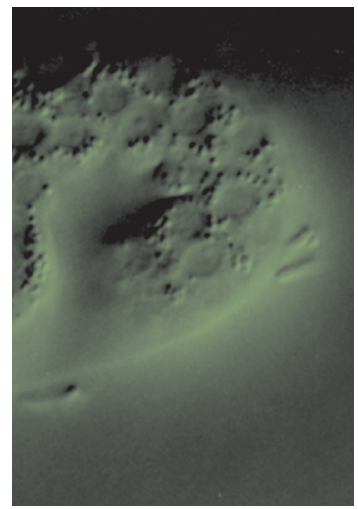

Centre d'Immunologie de Marseille Luminy, Cnrs UMR 6102, Inserm U. 136, Université de la Méditerranée, Case 906,

13288 Marseille Cedex 09,

France.

ewbank@ciml.univ-mrs.fr

système de reconnais-

sance chimiosensorielle. Ce comportement est relayé par des circuits neuronaux simples. Ces relais électriques ont d'ailleurs permis de modéliser le déplacement dans l'espace d'un robot qui mime le comportement du ver [2]. Concernant la perception des substances chimiques, le nématode peut détecter une gamme très large de molécules inorganiques et organiques solubles, telles que les ions $\left(\mathrm{Cl}^{-}, \mathrm{Na}^{+}, \mathrm{K}^{+}\right)$, les acides aminés, les nucléotides cycliques, et des substances odorantes volatiles telles que les alcools, les pyrazines et les thiazoles. On imagine que certaines combinaisons de ces molécules permettent la reconnaissance de divers micro-organismes. Sur les 302 neurones du nématode, une trentaine est impliquée dans la reconnaissance olfactive de composés chimiques. Certains neurones sont nécessaires au nématode pour sentir des composés attractifs, comme la paire de neurones AWA, d'autres pour sentir des composés répulsifs, comme la paire AWB. Les gènes codant pour plus de 700 récepteurs olfactifs présomptifs ont été identifiés dans le génome et, contrairement aux mammifères, où chaque neurone olfactif exprime majoritairement un seul récepteur, chez le ver plusieurs récepteurs sont présents sur chaque neurone chimiosensoriel [3]. La réponse d'attraction ou de répulsion du ver en direction d'un stimulus chimique n'est pas strictement liée à 
l'identité du récepteur. En effet, le récepteur ODR-10, qui est nécessaire à l'attraction vers le diacétyl, est normalement exprimé dans les neurones «d'attraction» AWA. Mais quand ODR-10 est exprimé de façon ectopique dans les neurones «de répulsion» $A W B$, le diacétyl devient un composé répulsif, suggérant un rôle clé du contexte cellulaire (Figure 1A) [4]. Par ailleurs, des tests comportementaux montrent que les vers sont capables de distinguer différents odorants. Les deux neurones $A W C$, qui sont structurellement similaires, peuvent détecter simultanément au moins cinq odorants attractifs, et la capacité de détecter et de reconnaître deux de ces odorants est associée avec l'expression d'au moins un récepteur olfactif, STR-2 (seven transmembrane receptor) dans un seul des neurones AWC, expression dite asymétrique (Figure 1B). Quand cette expression asymétrique est perdue, les vers ne peuvent plus distinguer le pentanedione du butanone. Cela montre que la discrimination des odeurs passe par la ségrégation de la détection dans des neurones distincts. L'expression asymétrique de STR-2 requiert l'activité d'une cascade de signalisation de type MAP kinase (mitogen activated protein kinase) impliquant SEK-1 et NSY-1. SEK-1 est une MAP2K homologue de MKK3/MKK6 chez les mammifères, alors que NSY- 1 est une MAP3K homologue de ASK1. Chez les mutants sek-1 ou nsy-1, STR-2 est exprimé dans les deux neurones de la paire AWC $[5,6]$. Mais même en l'absence d'une expression asymétrique de STR-2, les vers ne perdent pas la capacité de reconnaître toutes les odeurs. L'équipe de C. Bargmann a proposé que la discrimination puisse être traduite par des combinaisons uniques de différents récepteurs dans les neurones olfactifs spécifiques [7].

Depuis plusieurs années, l'existence d'une communication entre des vers par des phéromones produites constitutivement a été démontrée. En l'absence de nourriture ou lorsque la densité de population est élevée, de jeunes vers répondent aux phéromones en entrant dans un état de diapause (arrêt du développement) qui leur permet d'attendre des conditions meilleures [8]. Plus récemment, l'existence d'une phéromone produite exclusivement par des hermaphrodites pour l'attraction des mâles a été proposée [9]. Ces comportements sensoriels d'attraction sont relayés par les organes chimiosensoriels de la tête, les amphides. Concernant la répulsion, les organes chimiosensoriels de la queue, les phasmides, jouent un rôle modulateur négatif [10]. Cette perception sensorielle en deux points diamétralement opposés permet au nématode d'établir des repères stéréotypiques de son environnement chimique pour naviguer dans la complexité de son habitat.

Ajouté au fait d'être capable de détecter chimiquement des bactéries, les vers peuvent aussi les sentir physi- quement: arrivés dans une région riche en bactéries, les vers ralentissent leur allure, via un processus mécanosensoriel dépendant de la dopamine. À ce système s'ajoute une voie sérotoninergique qui module la réponse en fonction de l'expérience précédente du ver, qu'il ait été en carence ou bien nourri [11]. Dans la nature, ces deux ajustements du comportement pourraient permettre à un ver d'ingérer des bactéries d'une façon efficace en fonction de ses besoins. Les expériences de carence alimentaire modulent également l'expression des récepteurs chimiosensoriels et par conséquent le comportement olfactif du ver [12]. Même avec 302 neurones, il est possible d'apprendre!

\section{Ingestion-Digestion}

Quand le ver a trouvé sa pitance, il l'ingère via le pharynx, qui est un organe composé de neurones, de muscles et de cellules épithéliales, et d'une structure terminale appelée broyeur permettant de casser les

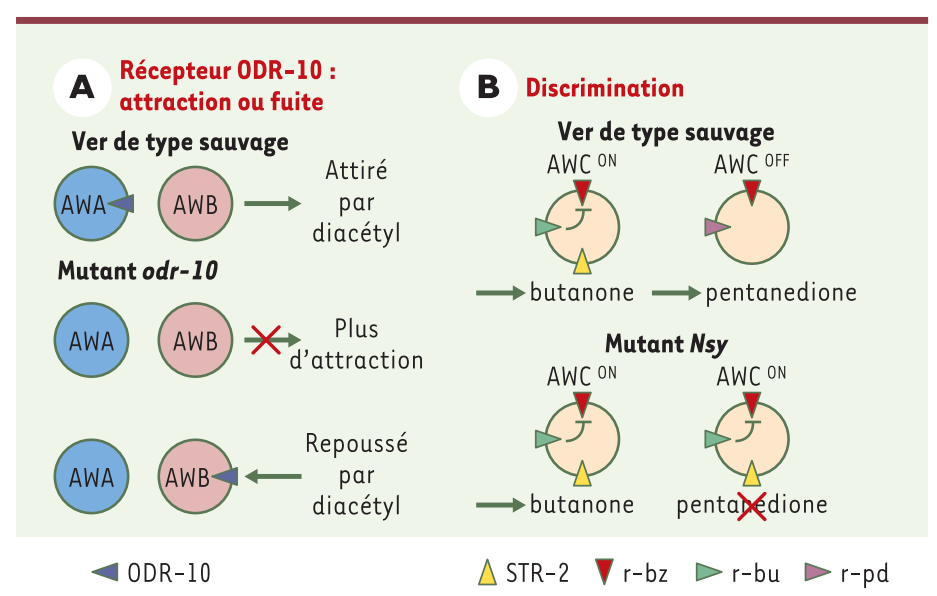

Figure 1. A. La reconnaissance olfactive de composés chimiques est liée au contexte cellulaire. Chez le ver sauvage, la présence du récepteur ODR-10 dans les neurones AWA confère un comportement d'attraction vers le diacétyl. En l'absence de ce récepteur dans un mutant odr-10, les vers ne sont plus attirés vers le diacétyl. Lorsqu'on exprime le récepteur de façon ectopique dans les neurones AWB en contexte mutant odr-10, les vers changent leur comportement et sont repoussés par le diacétyl. $\boldsymbol{B}$. Un modèle pour la discrimination. Chez le ver sauvage, les deux neurones $A W C$, qui sont structurellement et fonctionnellement similaires, reconnaissent le benzaldéhyde, mais un seul de ces deux neurones, $A W C^{O N}$, exprime le récepteur STR-2. AWCON reconnaît le butanone, AWCoFf reconnaît le pentanedione. La signalisation par le butanone atténue la signalisation par le benzaldéhyde dans AWC ${ }^{\circ}$. Dans le mutant nsy-1, le neurone AWC ${ }^{\text {OFf }}$ est transformé en $A W C^{\circ}$, l'attraction vers le pentanedione est perdue, et le butanone atténue la signalisation par le benzaldéhyde dans les deux neurones. $r$-bz, r-bu et r-pd sont respectivement les récepteurs présomptifs pour le benzaldéhyde, le butanone et le pentanedione (schéma adapté du modèle proposé dans [7]). 
bactéries (Figures $2 A$ et 2B). Les bases électrophysiologiques du mécanisme de pompage par le pharynx sont connues grâce aux travaux remarquables d'Avery et al. qui ont combiné des approches génétiques et le développement de méthodes de microdissection permettant d'obtenir des pharynx isolés et d'enregistrer des «électropharyngéogrammes » [13]. De récents résultats, utilisant la technique de FRET (fluorescence resonance energy transfer) avec une protéine dite «caméléon», ont permis de révéler les flux calciques associés aux contractions musculaires (Figure 2D) [14]. Les fibres

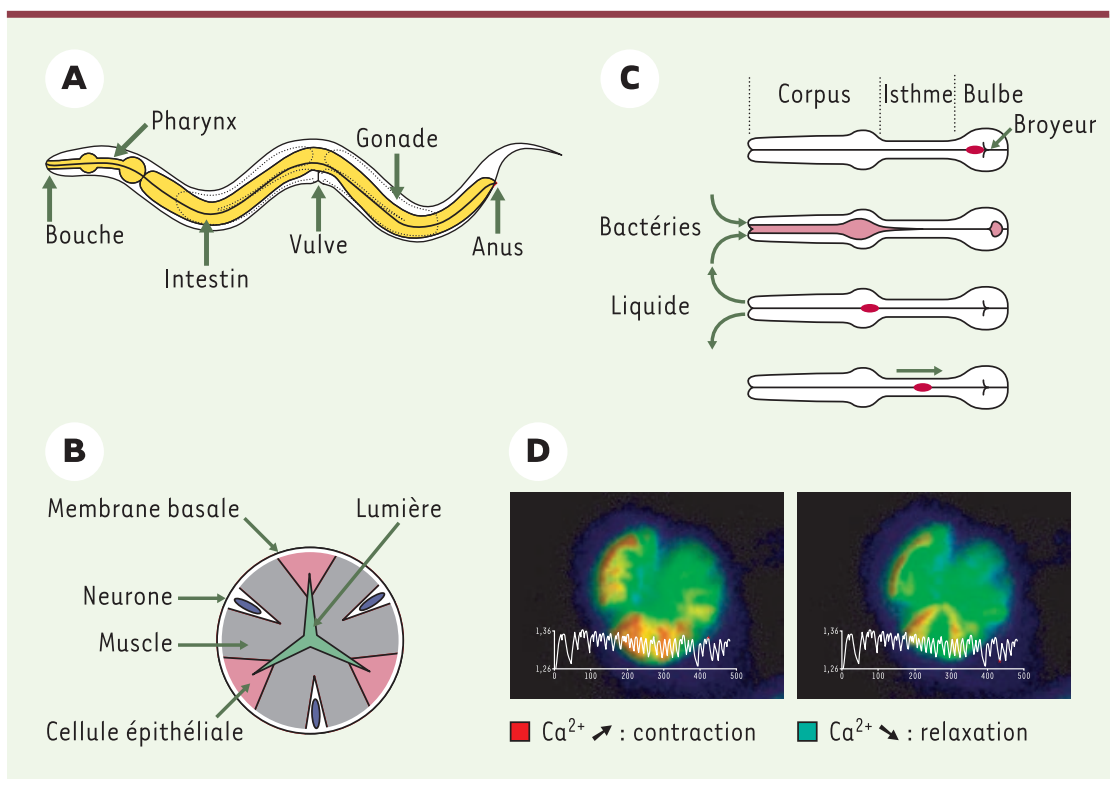

Figure 2. A. Schéma simplifié représentant les organes majeurs du ver. B. Coupe transversale du pharynx. Trois cellules musculaires arrangées autour de la lumière ont des fibres radiales, permettant l'ouverture du pharynx. Avec les trois cellules épithéliales, elles constituent un épithélium d'une épaisseur cellulaire qui sépare l'environnement de l'intérieur aqueux du ver. Le système nerveux pharyngial est physiquement séparé du système nerveux du ver par la membrane basale entourant tout le pharynx. C. Pompage et péristaltisme. Un cycle de pompage est composé d'une contraction presque simultanée du corpus, de l'isthme antérieur et du bulbe terminal, permettant l'ouverture de la lumière et l'absorption du liquide de l'environnement chargé de bactéries. La contraction du bulbe terminal inverse le broyeur, cassant ainsi les bactéries se trouvant devant le broyeur et entraînant les débris produits dans l'intestin. La relaxation suivante permet une nouvelle inversion du broyeur, et la fermeture de la lumière, expulsant ainsi le liquide. Le cycle est terminé par une contraction péristaltique de l'isthme postérieur qui transporte les bactéries du milieu de l'isthme au broyeur (parties B et C adaptées de [59] avec permission). D. Imagerie calcique révélant les contractions et relaxations du pharynx. L'indicateur protéique caméléon est composé de la protéine CFP (cyan fluorescent protein) fusionnée à la calmoduline et d'un domaine de fixation à la calmoduline (M13) fusionné à la protéine YFP (yellow fluorescent protein). À faible concentration de calcium, la calmoduline n'est pas fixée à M13, la fluorescence d'émission est celle de la CFP seule (en vert). En présence de calcium, la calmoduline se fixe à $M 13$, permettant une proximité entre les protéines CFP et YFP, et un transfert d'énergie d'excitation (FRET) entre les deux fluorochromes. La fluorescence d'émission résultante sera celle de la YFP (en rouge) (partie D adaptée de [14] avec permission). musculaires du pharynx étant orientées de façon radiale, leur contraction ouvre le lumen, permettant ainsi une aspiration du liquide extérieur. La contraction des muscles du bulbe terminal ferme les trois lobes du broyeur et provoque la destruction mécanique des bactéries. Alors que les débris solides sont filtrés dans l'intestin, le liquide restant est expulsé avant le prochain cycle de contractions (Figure 2C).

Le broyeur n'est pas indispensable à la survie de C. elegans, car l'intestin est le site de la synthèse d'enzymes qui contribuent à la dégradation des bactéries, telles que les protéases aspartiques et cystéisozymes [15]. Le tube digestif est anatomiquement simple (Figure 3A), mais montre une spécialisation en terme de régionalisation de l'expression de gènes [16]. Le transit des aliments se fait passivement du pharynx à l'anus. Comme le contenu de l'intestin est entièrement vidé chaque minute grâce à la défécation, les processus d'absorption de nutriments, ainsi que les activités digestives, doivent être extrêmement rapides.

Chaque cycle de défécation est constitué de trois mouvements musculaires (Figure 3B). Une première contraction des muscles postérieurs du corps envoie le contenu de l'intestin vers la tête. Elle est suivie d'une contraction des muscles antérieurs, puis, immédiatement après, de la contraction simultanée des muscles postérieurs de l'intestin, du sphincter anal et du dépresseur anal permettant la sortie des déchets. Ce cycle est contrôlé de façon non autonome par un récepteur inositol triphosphate, qui règle le relargage de calcium dans les cellules intestinales de façon cyclique [17]. Ce modèle simple a donc donné l'une des premières indications de la nature moléculaire d'une horloge biologique.

\section{Les cycles}

À l'instar de la fréquence de défécation, plusieurs autres comportements sont très réguliers: le pompage, à raison de trois cycles par seconde, ou la ponte, de six œufs par heure (à $\left.20^{\circ} \mathrm{C}\right)$. Même si leur gamme de fréquences est variée, ces cycles comportementaux sont tous contrôlés d'une façon coordonnée chez les vers de type sauvage. Ces rythmes 
varient tous en fonction de la température: plus celleci diminue, plus les rythmes sont lents. Inversement, plus la température augmente, plus les rythmes sont rapides, jusqu'à une température seuil entre $22^{\circ} \mathrm{C}$ et $25^{\circ} \mathrm{C}$ à laquelle les cycles n'augmentent plus. Cette absence de compensation de la température ces cycles, appelés ultradiens, contraste avec l'indépendance de la température des cycles circadiens. Ces derniers sont connus depuis plusieurs années chez de multiples espèces mais n'ont été mis en évidence chez le ver que récemment $[18,19]$. Comme pour la plupart des espèces, on imagine que la lumière est le stimulus qui entraîne le cycle circadien chez C. elegans. Mais si le travail de A.H. Burr en 1985 avait montré que le ver est $(\rightarrow) \mathrm{m} / \mathrm{s}$ p. 474 $1997, \mathrm{n}^{\circ} 4$, réceptif à la lumière [20], les bases moléculaires et physiologiques de la perception de la lumière n'ont pas encore été établies.

Une classe de mutants «clk» (clock) présente un ralentissement des comportements rythmiques ultradiens. À ce jour, le mutant $c l k-1$ qui se développe également plus lentement, est le mieux caractérisé. Chez le ver sauvage, la durée du développement embryonnaire est respectivement de 13,3 heures et 23,6 heures à $20^{\circ} \mathrm{C}$ et à $15^{\circ} \mathrm{C}$. Or, pour le mutant clk-1(qm30) les durées correspondantes sont de 22,8 et 46,1 heures. Divers phénotypes mutants clk-1 semblent être connectés. Ainsi, les individus $c l k-$ 1 ayant le développement embryonnaire le plus lent ont un cycle de défécation relativement plus rapide que les individus mutants qui se développent plus rapidement, et inversement. En outre, contrairement au ver sauvage, les mutants $c l k-1$ ont perdu la capacité d'adapter rapidement la vitesse de développement ou de comportement lors d'une variation abrupte de température (Figure 4). Ces résultats ont conduit l'équipe d'Hekimi à proposer un mécanisme de contrôle temporel général chez le ver (pour revue, voir [21]) $(\rightarrow)$ qui a été confirmé par des études plus récentes [22].

Le gène $c l k-1$ code pour l'enzyme qui catalyse l'avantdernière étape de la synthèse d'ubiquinone [23, 24]. L'ubiquinone, aussi appelé co-enzyme $Q$, ou simplement ' $Q$ ', est un co-facteur lipidique présent dans toutes les membranes cellulaires jouant un rôle dans de nombreuses réactions d'oxydo-réduction, y compris dans la production d'ATP par la chaîne respiratoire des mitochondries. Les mutants $c l k-1$ ne synthétisent pas $\rho$ mais accumulent la déméthoxyubiquinone (DMQ), un précurseur bio-synthétique, qui peut se substituer à $\rho$ dans les mitochondries. Hors des mitochondries, $\rho$ est indispensable durant le développement, rôle qui ne peut être rempli par DMQ. Dans les conditions normales de culture, les mutants $c l k-1$ peuvent se développer en assimilant $\rho$ des bactéries. En l'absence totale de $\rho$, il n'y a pas de développement [25]. Chez C. elegans, le rôle précis de $Q$ hors des mitochondries n'est pas connu, ni le lien entre le changement du niveau de $Q$, la vitesse du développement et les phénotypes comportementaux des mutants $c l k-1$ [26].

\section{Durée de vie}

Les mutants $c l k-1$ vivent également $25 \%$ plus longtemps que les vers sauvages, qui ont une durée de vie moyenne de 20 jours à $20^{\circ} \mathrm{C}$. Un facteur limitant la durée de vie est l'accumulation de dommages oxydatifs dus aux dérivés réactifs de l'oxygène (reactive oxygen species ou ROS). Un des sites principaux de la production des ROS est la mitochondrie, et cette production varie en fonction de la vitesse et de l'efficacité du transport des électrons. Dans le cas des mutants clk-1, l'augmentation de la durée de vie pourrait être due à l'altération du transport des électrons provoquant une diminution de la production de ROS (Figure 5). De façon significative, un mutant dans le gène isp- 1 qui code pour la protéine fersulfure Rieske du complexe III mitochondrial a un transport d'électrons réduit [27] et une durée de vie de $50 \%$ plus longue que celle des vers sauvages.

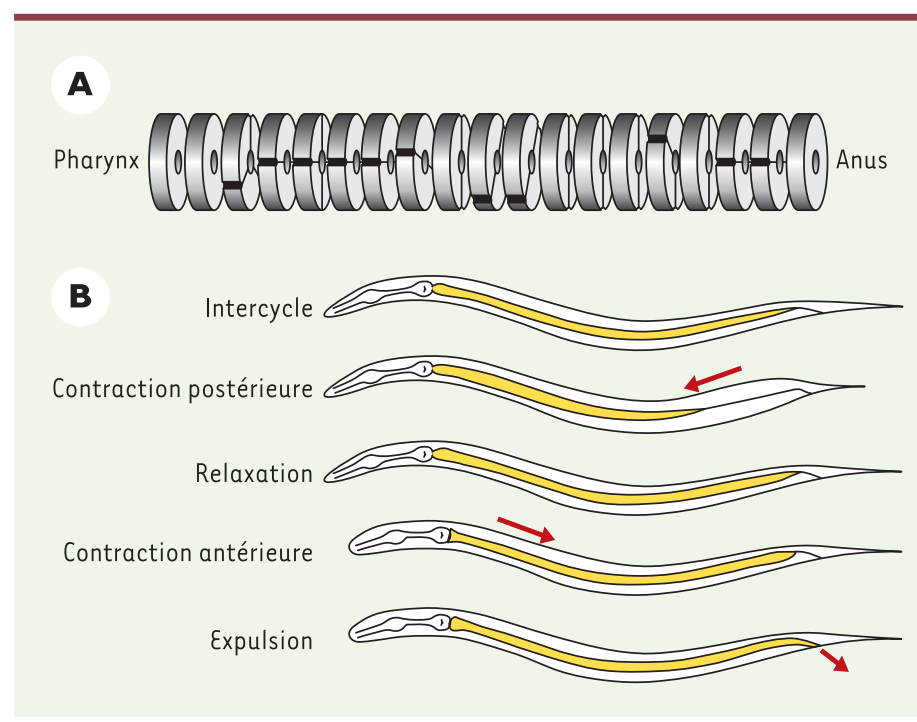

Figure 3. A. Schéma représentant la structure de l'intestin. L'intestin est un tube, formé d'une seule couche de cellules. Il est composé d'une série de toroïdes, constitués chacun d'une à quatre cellules polyploïdes. B. Cycle de défécation. La durée d'un cycle est inférieure à une minute. Un cycle commence par une contraction des muscles postérieurs du corps entraînant le contenu de l'intestin vers l'avant. Après une courte période de relaxation, une contraction des muscles antérieurs du corps reflue le contenu de l'intestin vers l'arrière. À cette contraction s'ajoute la contraction des muscles entériques permettant l'ouverture de l'anus et l'expulsion du contenu de l'intestin (adapté de [59] avec permission). 
Une deuxième classe de vers mutants, appelée daf à durée de vie augmentée, implique une voie de signalisation analogue à la voie insuline/IGF (insulin-like growth factor) chez les vertébrés. Ainsi, le gène daf-2 code pour un récepteur de l'insuline. Si les mutants daf-2 ont une durée de vie moyenne de 34 jours, les doubles mutants daf-2 clk-1 vivent 5 fois plus longtemps que les vers sauvages, suggérant que les deux gènes fonctionnent dans des voies différentes. De plus, l'activité du facteur de transcription DAF-16, qui est contrôlée par la voie daf-2, est indépendante de clk-1 [28]. Deux hypothèses non exclusives sont avancées pour expliquer le rôle de la voie insuline dans la longévité: soit les vers seraient plus résistants aux stress oxydatifs, soit ils présenteraient une altération métabolique réduisant la production de ROS. Dans le dernier cas, les voies daf et clk-l convergeraient au niveau des mitochondries.

L'équipe de C. Kenyon a récemment montré que des mutants dans le gène mes- 1 , qui code pour un récepteur impliqué dans une voie Src, où la lignée germinale est absente, vivent plus longtemps [29]. De même pour des mutants dans le gène $g l p-1$, qui code pour un récepteur Notch nécessaire à la prolifération de la lignée germinale. La durée de vie des mutants glp-1 thermosensibles, chez lesquels l'ensemble des cellules souches de la lignée germinale est dirigé vers la méiose, est aussi rallongée. À l'inverse, des mutants chez lesquels la lignée germinale prolifère de façon excessive vivent moins longtemps, suggérant que l'augmentation de la durée de vie est liée à l'absence de prolifération et non à la différenciation de la lignée germinale. De plus,

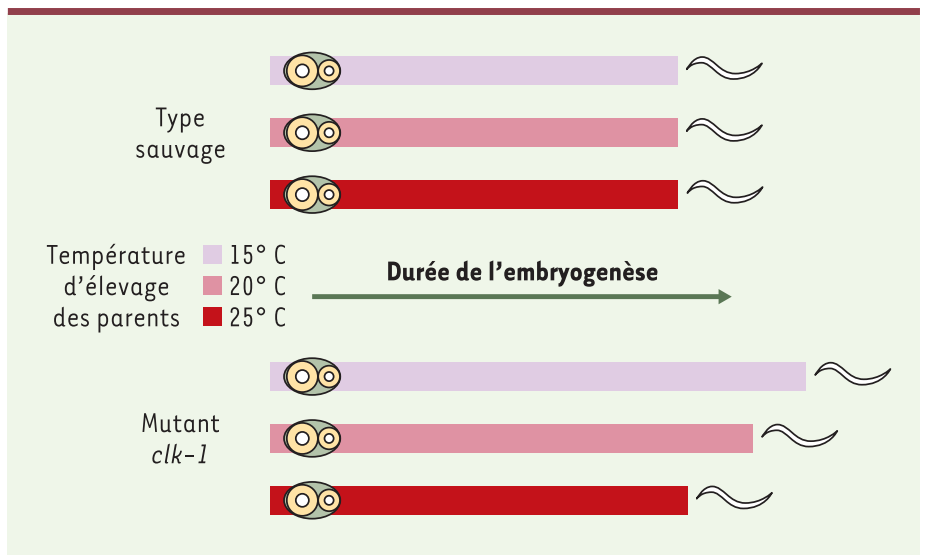

Figure 4. Influence de la température sur la vitesse de développement. Représentation schématique d'une expérience dans laquelle des embryons et leurs parents ont été élevés à trois températures différentes: $15^{\circ} \mathrm{C}, 20^{\circ} \mathrm{C}$ et $25^{\circ} \mathrm{C}$. Tous les embryons ont ensuite été transférés à $20^{\circ} \mathrm{C}$ pour le restant de l'embryogenèse et la durée jusqu'à l'éclosion a été mesurée. Pour le type sauvage, ce traitement n'affecte pas la durée de l'embryogenèse. En revanche, la durée de l'embryogenèse des mutants dépend de la température d'élevage avant le transfert (adapté de [21]). l'augmentation de la longévité des mutants sans lignée germinale est dépendante de daf-16, suggérant une contribution de la voie insuline/IGF [29] et, inversement, l'augmentation de la longévité des mutants clk1 est dépendante de la présence de la gonade [22], liant $\rho$ et la lignée germinale. Pour une discussion plus détaillée à propos de ces interactions et d'autres facteurs contrôlant la longévité chez $C$. elegans, comme les phénomènes de silencing [30] et le maintien de la longueur des télomères [31], les lecteurs peuvent consulter des revues spécialisées [32-34], ainsi que des articles récents sur des études systématiques par l'interférence par ARN double brin (ARN) sur la longévité $[22,35]$.

\section{Maladies et mécanismes de défense}

Dans la nature, les vers ne vivent probablement pas jusqu'à leur limite physiologique car les conditions environnementales sont loin d'être idéales, les vers étant notamment confrontés à de multiples pathogènes potentiels [15]. Certains, comme le champignon Drechmeria coniospora [36] ou la bactérie à Gram positif Microbacterium nematophilum [37], semblent être spécifiques des nématodes. Mais les vers sont également sensibles à de nombreuses bactéries normalement présentes dans le sol, comme certaines espèces d'Agrobacterium, d'Erwininia [38], de Pseudomonas [39] et de Serratia [40]. Ces pathogènes ont des modes d'infection différents et provoquent des symptômes distincts. Dans le cas du champignon $D$. coniospora, des spores adhèrent à la cuticule et envoient des hyphes à l'intérieur du ver via toutes les ouvertures (amphides, vulve, anus...). Certaines bactéries comme M. nematophilum provoquent une infection cuticulaire, tout en restant à l'extérieur du ver. D'autres, comme S. marcescens, sont capables de forcer leur passage jusqu'à l'intestin. À ce jour, l'interaction la mieux caractérisée est celle entre $C$. elegans et $P$. aeruginosa. Selon la souche ou les conditions de culture, cette bactérie peut tuer le ver, soit par empoisonnement au cyanide [41], soit par production d'autres toxines agissant via la production de radicaux libres, soit encore par un processus infectieux [39].

Nous avons évoqué ci-dessus la capacité qu'ont les vers de distinguer différents produits chimiques volatils ou solubles. Dans la nature, cette capacité est probablement essentielle à leur survie. Les vers sont en effet capables de discriminer différents micro-organismes et même d'éviter certains d'entre eux. La capacité des vers d'éviter $S$. marcescens est dépendante de l'homologue de la protéine Toll [42]. Cette famille de récepteurs trans-membranaires est associée à la reconnaissance de pathogènes, à la fois chez la drosophile et les 
vertébrés où les protéines Toll sont responsables du déclenchement des défenses anti-microbiennes $(\rightarrow)$.

$(\rightarrow) \mathrm{m} / \mathrm{s}$ 2000, $\mathrm{n}^{\circ} 12$, p. 1439 Leur activation conduit à la translocation nucléaire d'un facteur de transcription de la famille NF-KB, et à l'induction d'expression de gènes de défense [43]. Chez le ver, Toll ne semble pas être impliquée dans des défenses inductibles, et aucun homologue de NF-KB n'a pu être identifié dans la séquence génomique du ver [42].

Des défenses inductibles existent néanmoins chez le ver. $\varepsilon n$ effet, des analyses différentielles par puces à ADN ont montré que plusieurs gènes sont induits de façon spécifique au cours de l'infection de C. elegans par S. marcescens. Outre les gènes spécifiques du ver sans fonction connue, plusieurs lectines sont induites, et l'on sait que certaines lectines jouent des rôles clés dans l'immunité innée chez les vertébrés et les invertébrés [44, 45]. L'expression de certains lysozymes est également induite après infection, et la surexpression de l'un d'entre eux augmente la survie du ver infecté par une souche de $S$. marcescens. Certains des gènes induits sont sous le contrôle de la voie TGF $\beta$ et, de façon significative, le mutant $d b l-1 /$ TGF $\beta$ est lui-même plus sensible aux infections bactériennes [16].

L'équipe de F.M.Ausubel a complété ces études par une approche génétique en identifiant des mutants de $C$. elegans hypersensibles à l'infection par $P$. aeruginosa. Cette équipe a non seulement montré que plusieurs mutants de la voie $d b l-1 /$ TGF $\beta$ sont plus sensibles à cette bactérie [46], mais aussi qu'une voie MAP kinase est également impliquée dans les défenses anti-bactériennes [47]. En effet, après criblage, deux mutants hypersensibles à l'infection, sek-l et nsy1 , codant (comme cela est décrit ci-dessus) respectivement pour une MAP2K et une MAP3K, ont été identifiés. II est curieux que ces deux gènes soient impliqués à la fois dans la spécification d'une identité cellulaire dans une paire de neurones chimiosensoriels et dans les défenses anti-microbiennes. Dans le contexte de la résistance à l'infection, une nouvelle cible de cette voie a été identifiée par inactivation par ARN, la MAPK PMK-1 de la famille p38. La même voie MAPK (NSY-1/SEK-1/PMK-1) est aussi requise pour la résistance de $C$. elegans à l'arsenic, suggérant qu'elle puisse faire partie d'un système plus général de résistance au stress.

L'équipe de F.M.Ausubel a également montré l'implication de l'apoptose dans les défenses de $C$. elegans contre la bac- térie Salmonella typhimurium [48]. Plus récemment, cette équipe a pu démontrer un lien fonctionnel entre la voie NSY-1/SEK-1/PMK-1 et l'apoptose [49]. L'ensemble de ces données indique que l'immunité innée du ver présente une complexité moléculaire qui reste encore à élucider [50].

\section{Nouvelles technologies}

L'achèvement du séquençage du génome du ver il y a 4 ans [51] a marqué le départ de nouvelles investigations, permettant par exemple la fabrication de puces à ADN contenant 18000 gènes, soit $95 \%$ du génome de $C$. elegans. À l'heure actuelle, plus de 500 expériences ont été effectuées à Stanford ('́tats-Unis) avec ces puces à ADN dans diverses conditions: par exemple des vers sauvages ou mutants, pris à différents stades de développement, ou élevés dans des conditions différentes (stress, irradiation...). En corrélant toutes ces expériences, les gènes présentant une même variation d'expression dans l'ensemble des conditions testées se retrouvent ainsi groupés et sont visualisés sur une carte topographique en montagnes d'expression (Figure 6). Quarante-quatre montagnes ont été identifiées, et l'ensemble de ces données est en libre accès dans la base de donnée Wormbase. Plusieurs groupes de gènes dans différentes montagnes ont été analysés par les auteurs,

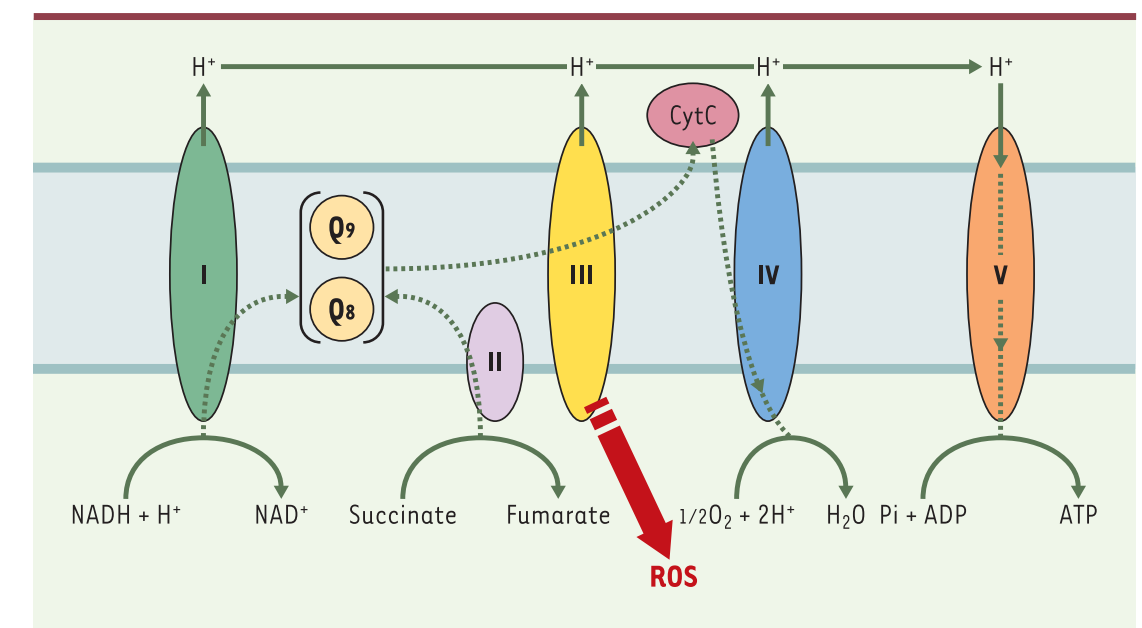

Figure 5. Chaîne de transport des électrons. Cette chaîne est constituée de cinq complexes protéiques I, II, III, IV, V (NADH- $\rho$ réductase, succinate- $\rho$ réductase, $Q$-cytochrome c réductase, cytochrome coxydase, ATP synthase). Le coenzyme $\rho(\rho)$ est un facteur lipidique soluble crucial pour le transport des électrons à travers la membrane interne de la mitochondrie, nécessaire à la synthèse d'ATP. Les vers synthétisent l'isoforme $Q_{9}$ et peuvent aussi obtenir l'isoforme $Q_{8}$ synthétisée par les bactéries. Le transport des électrons, surtout au niveau du complexe III, produit des dérivés réactifs de l'oxygène (ROS). Un régime pauvre en $\rho$ chez les vers allongerait la durée de vie en réduisant la production des ROS lors du transport des électrons. De même, chez un

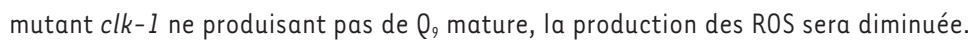


confirmant une corrélation de fonction ou de localisation tissulaire. Cette carte d'expression peut permettre de prédire, dans une certaine mesure, une fonction pour un gène ou un groupe de gènes inconnus [52].

L'obtention de la séquence génomique offre la possibilité d'entreprendre d'autres approches à grande échelle. Le projet ORFéome a cloné plus de $60 \%$ des ADNc complets du nématode sur les quelque 19000 gènes prédits dans des vecteurs navettes à sorties multiples, permettant notamment des expériences de double hybride à grande échelle [53, 54]. Par la technique d'ARNi, des cribles systématiques ont déjà produit des résultats $[55,56](\rightarrow)$. Actuellement, plusieurs groupes conduisent des cribles par ARN pour identifier des gènes présentant des phénotypes synthétiques*, c'est-à-dire seulement dans le contexte d'un fond génétique mutant spécifique, pour révéler des redondances fonctionnelles ou gènes qui agissent dans une même voie ou un même processus biologique. Mais I'ARN présente certains inconvénients, comme la varia-

* Phénotype d'un double mutant lorsque les simples mutations sont silencieuses. bilité des phénotypes d'un individu à l'autre et l'absence de transmission héréditaire. D'où l'intérêt de développer des méthodes de recombinaison homologue chez le ver. Un premier pas a été fait avec la mise au point d'une méthode de mutagenèse par transposition hétérologue. Décrite pour la première fois par J.L. Bessereau et al., cette méthode est rapidement devenue un outil de choix, en raison de la rapidité de clonage des gènes à l'issue de la mutagenèse [57]. Mais l'inconvénient de cette méthode est l'inefficacité relative de la mutagenèse, qui nécessite de réaliser des cribles à grande échellle. L'existence d'un trieur automatique de ver, similaire à celui décrit pour la drosophile [58] pourrait pallier cet inconvénient. Un tel trieur vient d'être installé au Génopole de Marseille (http: //www.ciml.univmrs.fr/EWBANK_jonathan/Genopole.html) et va être utilisé en tant que plate-forme d'analyse pour la communauté scientifique française travaillant sur $C$. elegans. L'utilisation de ces outils fait de $C$. elegans un modèle de choix pour la génomique fonctionnelle et contribuera à élargir nos connaissances concernant les bases cellulaires et moléculaires de la physiopathologie. $\diamond$ $(\rightarrow) \mathrm{m} / \mathrm{s}$

$2001, n^{\circ} 3$,

p. 355

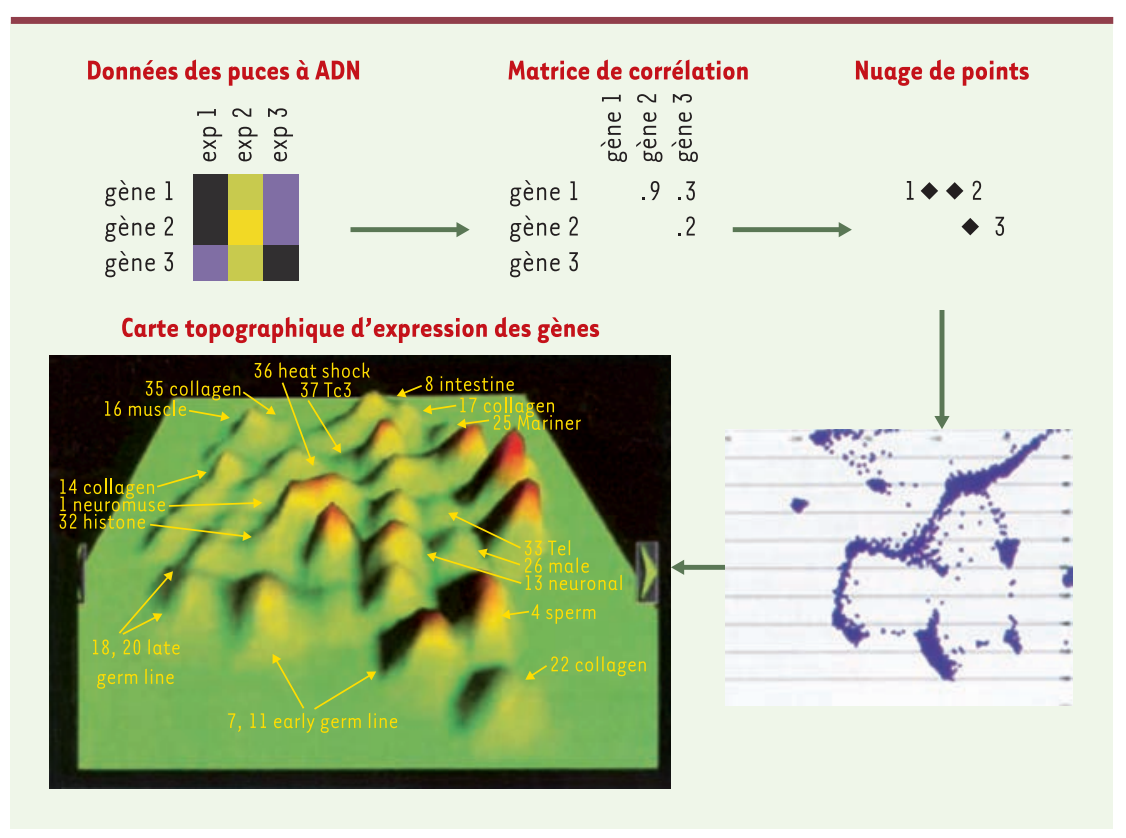

Figure 6. Construction d'une carte topographique d'expression de gènes. Dans la matrice d'expression, le jaune représente l'augmentation relative de l'expression d'un gène et le bleu une diminution. Trois gènes et trois expériences sont donnés en exemple. Les données d'expression sont utilisées pour calculer un indice de corrélation entre chaque paire de gènes. Les gènes avec le plus fort taux de corrélation sont ensuite utilisés pour construire un nuage de points en $2 D$. Ce graphe est alors converti en une carte topographique d'expression de gènes représentant les corrélations en 3D, où l'altitude correspond à la densité des gènes (reproduit de [60]) $(\rightarrow)$.

\section{SUMMARY \\ A worm's life}

Despite its relative anatomic simplicity, the nematode Caenorhabditis elegans (C. elegans) is a complex multicellular organism. In this review, we describe studies that have contributed to a better understanding of certain aspects of the worm's physiology. We focus on the cellular and molecular basis of the interaction between $C$. elegans and its environment, including its sensory capacities, the intrinsic biological clock that governs the speed of its life, and on some of the factors that control its life span. We also outline very recent findings that have demonstrated the existence of an innate immune system in C. elegans. Finally, we highlight a number of novel techniques that are transforming the worm from a largely genetic model system into an attractive organism for functional genomic studies. $\diamond$

$(\rightarrow) \mathrm{m} / \mathrm{s}$ 2002, $n^{\circ} 1$, p. 97 


\section{RÉFÉRENCES}

1. Riddle DL, Blumenthal T, Meyer BJ, Priess JR. C. elegans II. Woodbury: Cold Spring Harbor Laboratory Press, 1997: 1222 p.

2. Morse TM, Ferree TC, Lockery SR. Robust spatial navigation in a robot inspired by C. elegans. Adaptive Behav 1998; 6: 391-408.

3. Troemel ER, Chou JH, Dwyer ND, Colbert HA, Bargmann $\mathrm{Cl}$. Divergent seven transmembrane receptors are candidate chemosensory receptors in C. elegans. Cell 1995; 83: 207-18.

4. Troemel $\varepsilon R$, Kimmel BE, Bargmann $\mathrm{Cl}$. Reprogramming chemotaxis responses: sensory neurons define olfactory preferences in C. elegans. Cell 1997; 91: 161-9.

5. Sagasti A, Hisamoto $N$, Hyodo J, Tanaka-Hino M, Matsumoto K, Bargmann C. The CaMKII UNC-43 activates the MAPKKK NSY1 to execute a lateral signaling decision required for asymmetric olfactory neuron fates. Cell 2001; 105: 221-32.

6. Tanaka-Hino M, Sagasti A, Hisamoto $N$, et al. SEK-1 MAPKK mediates $\mathrm{Ca}^{2+}$ signaling to determine neuronal asymmetric development in Caenorhabditis elegans. EMBO Rep 2002; 3: 56-62.

7. Wes PD, Bargmann C. C. elegans odour discrimination requires asymmetric diversity in olfactory neurons. Nature 2001; 410: 698-701.

8. Golden JW, Riddle DL. The Caenorhabditis elegans dauer larva: developmental effects of pheromone, food, and temperature. Dev Biol 1984; 102: 368-78.
9. Simon JM, Sternberg PW. Evidence of a mate-finding cue in the hermaphrodite nematode Caenorhabditis elegans. Proc Natl Acad Sci USA 2002; 99: 1598-603.

10. Hilliard MA, Bargmann Cl, Bazzicalupo P. C. elegans responds to chemical repellents by integrating sensory inputs from the head and the tail. Curr Biol 2002; 12: 730-4.

11.Sawin $\varepsilon R$, Ranganathan $R$, Horvitz HR. C. elegans locomotory rate is modulated by the environment through a dopaminergic pathway and by experience through a serotonergic pathway. Neuron 2000; 26: 619-31.

12.Peckol EL, Troemel ER, Bargmann $\mathrm{Cl}$. Sensory experience and sensory activity regulate chemosensory receptor gene expression in Caenorhabditis elegans. Proc Natl Acad Sci USA 2001; 98: 11032-8.

13. Raizen DM, Avery L. Electrical activity and behavior in the pharynx of Caenorhabditis elegans. Neuron 1994; 12: 483-95.

14. Kerr R, Lev-Ram V, Baird G, Vincent P, Tsien Ry, Schafer WR. Optical imaging of calcium transients in neurons and pharyngeal muscle of $C$. elegans. Neuron 2000; 26: 583-94.

15.Ewbank JJ. Tackling both sides of the host-pathogen equation with Caenorhabditis elegans. Microbes Infect 2002; 4: 247-56.

16. Mallo GV, Kurz CL, Couillault $C$, et al. Inducible antibacterial defense system in C. elegans. Curr Biol 2002; 12: 1209-14.

17. Dal Santo P, Logan MA, Chisholm AD, Jorgensen EM. The inositol trisphosphate receptor regulates a 50 second behavioral rhythm in C. elegans. Cell 1999; 98: 757-67.
18. Kippert F, Saunders DS, Blaxter ML. Caenorhabditis elegans has a circadian clock. Curr Biol 2002; 12: R47-9.

19.Saigusa T, Ishizaki S, Watabiki S, et al. Circadian behavioural rhythm in Caenorhabditis elegans. Curr Biol 2002; 12: R46-7.

20.Burr AH. The photomovement of Caenorhabditis elegans, a nematode which lacks ocelli. Proof that the response is to light not radiant heating. Photochem Photobiol 1985; 41: 577-82.

21. Hekimi S. Une horloge cellulaire et physiologique règle la vie du nématode Caenorhabditis elegans. Med Sci 1997; 13: 474-82

22. Dillin A, Hsu AL, Arantes-Oliveira N, et al. Rates of behavior and aging specified by mitochondrial function during development. Science 2002; 298: 2398-401.

23. Ewbank JJ, Barnes TM, Lakowski B, Lussier M, Bussey $\mathrm{H}$, Hekimi S. Structural and functional conservation of the Caenorhabditis elegans timing gene clk-1. Science 1997; 275: 980-3.

24. Stenmark P, Grunler J, Mattsson J, Sindelar PJ, Nordlund P, Berthold DA. A new member of the family of di-iron carboxylate proteins. Coq7 (clk-1), a membrane-bound hydroxylase involved in ubiquinone biosynthesis. J Biol Chem 2001; 276: 33297-300.

25. Hihi AK, Gao Y, Hekimi S. Ubiquinone is necessary for Caenorhabditis elegans development at mitochondrial and nonmitochondrial sites. J Biol Chem 2002; 277: 2202-6.
26. Miyadera $H$, Kano K, Miyosh H, Ishii N, Hekimi S, Kita K. Quinones in long-lived clk1 mutants of Caenorhabditis elegans. FEBS Lett 2002; 512: 33-7.

27. Feng J, Bussiere F, Hekimi S. Mitochondrial electron transport is a key determinant of life span in Caenorhabditis elegans. Dev Cell 2001; 1: 633-44.

28.Lakowski B, Hekimi S. Determination of life-span in Caenorhabditis elegans by four clock genes. Science 1996; 272: 1010-3.

29. Arantes-Oliveira N, Apfeld J, Dillin A, Kenyon C. Regulation of life-span by germ-line stem cells in Caenorhabditis elegans. Science 2002; 295: 502-5.

30.Tissenbaum HA, Guarente L. Increased dosage of a sir-2 gene extends lifespan in Caenorhabditis elegans. Nature 2001: 410: 227-30.

31.Benard C, McCright B, Zhang y, Felkai S, Lakowski B, Hekimi S. The $C$. elegans maternal-effect gene $c l k-2$ is essential for embryonic development, encodes a protein homologous to yeast Tel $2 p$ and affects telomere length. Development 2001; 128: 4045-55.

32.Benard C, Hekimi S. Longlived mutants, the rate of aging, telomeres and the germline in Caenorhabditis elegans. Mech Ageing Dev 2002; 123: 869-80.

33.Partridge L, Gems D. Mechanisms of ageing: public or private? Nat Rev Genet 2002 ; 3 : 165-75.

34. Lithgow GJ, Walker GA. Stress resistance as a determinate of $C$. elegans lifespan. Mech Ageing Dev 2002; 123: 765-71.

35. Lee SS, Lee RY, Fraser AG, Kamath RS, Ahringer J, Ruvkun G. A systematic RNAi screen identifies a critical role for mitochondria in C. elegans longevity. Nat Genet 2003; $33: 40-8$. 
36.Jansson HB. Adhesion of conidia of Drechmeria coniospora to

Caenorhabditis elegans wild type and mutants. J Nematol 1994; $26: 430-5$.

37. Hodgkin J, Kuwabara PE, Corneliussen B. A novel bacterial pathogen, Microbacterium nematophilum, induces morphological change in the nematode $C$. elegans. Curr Biol 2000; 10: 1615-8.

38. Couillault C, Ewbank JJ. Diverse bacteria are pathogens of Caenorhabditis elegans. Infect Immun 2002: 70: 4705-7.

39.Tan MW, Ausubel FM. Caenorhabditis elegans: a model genetic host to study Pseudomonas aeruginosa pathogenesis. Curr Opin Microbiol 2000; 3: 29-34.

40. Kurz CL, Ewbank JJ. Caenorhabditis elegans for the study of host-pathogen interactions. Trends Microbiol 2000; 8: 142-4.

41.Gallagher LA, Manoil C. Pseudomonas aeruginosa PAO1 kills Caenorhabditis elegans by cyanide poisoning. J Bacteriol 2001; 183: 6207-14.

42. Pujol N, Link EM, Liu LX, et al. A reverse genetic analysis of components of the Toll signaling pathway in Caenorhabditis elegans. Curr Biol 2001; 11: 809-21.
43. Imler JL, Hoffmann JA. Signaling mechanisms in the antimicrobial host defense of Drosophila. Curr Opin Microbiol 2000; 3: 16-22.

44. Franc NC, White K. Innate recognition systems in insect immunity and development: new approaches in Drosophila. Microbes Infect 2000; 2: 243-50.

45. Linehan SA, MartinezPomares L, Gordon S. Macrophage lectins in host defence. Microbes Infect $2000 ; 2$ : 279-88.

46.Tan MW. Genetic and genomic dissection of host-pathogen interactions using a $P$. aeruginosa- $C$. elegans pathogenesis model. Pediatr Pulmonol $2001 ; 32: 96-7$.

47. Kim DH, Feinbaum R, Alloing $G$, et al. A conserved $p 38$ MAP kinase pathway in Caenorhabditis elegans innate immunity. Science 2002; 297: 623-6.

48. Aballay A, Ausubel FM. Programmed cell death mediated by ced- 3 and ced-4 protects Caenorhabditis elegans from Salmonella typhimurium-mediated killing. Proc Natl Acad Sci USA 2001; 98 : 2735-9.
49. Aballay A, Drenkard $\varepsilon$, Hilbun LR, Ausubel FM. Caenorhabditis elegans innate immune response triggered by Salmonella enterica requires intact LPS and is mediated by a MAPK signaling pathway. Curr Biol 2003; 13: 47-52.

50. Kurz CL, Ewbank JJ, Caenorhabditis elegans: an emerging model for the study of innate immunity. Nat Rev Genet 2003; 4: 380-90.

51. Genome sequence of the nematode $C$. elegans: a platform for investigating biology. The $C$. elegans Sequencing Consortium. Science 1998; 282: 2012-8.

52. Kim SK, Lund J, Kiraly M, et al. A gene expression map for Caenorhabditis elegans. Science 2001 293: 2087-92.

53. Reboul J, Vaglio P, Tzellas N et al. Open-reading-frame sequence tags (OSTs) support the existence of at least 17,300 genes in $C$. elegans. Nat Genet 2001; 2: 332-6.

54. Reboul J, Vaglio P, Rual JF, et al. C. elegans ORFeome version 1.1: experimental verification of the genome annotation and resource for proteome-scale protein expression. Nat Genet 2003 34: 35-41.
55.Pujol N, Ewbank JJ. C. elegans, du génome à l'invalidation systématique par interférence par ARN. Med Sci 2001; 17 : 355-7.

56. Kamath RS, Fraser AG, Dong $y$, et al. Systematic functional analysis of the Caenorhabditis elegans genome using RNAi. Nature 2003; 421: 231-7.

57. Bessereau JL, Wright $A$, Williams DC, Schuske K, Davis MW, Jorgensen EM. Mobilization of a Drosophila transposon in the Caenorhabditis elegans germ line. Nature 2001; 413: 70-4.

58. Furlong દદ, Profitt D, Scott MP. Automated sorting of live transgenic embryos. Nat Biotechnol 2001; 19: 153-6.

59.Avery L, Thomas JH. In: Riddle DL, Blumenthal T, Meyer BJ, Priess JR, eds. C. elegans II. Woodbury: Cold Spring Harbor Laboratory Press, 1997: 679716.

60. Kurz CL, Pujol N. C. elegans: des montagnes de données. Med Sci 2002; 18: $97-9$.
TIRÉS À PART J.J. Ewbank 